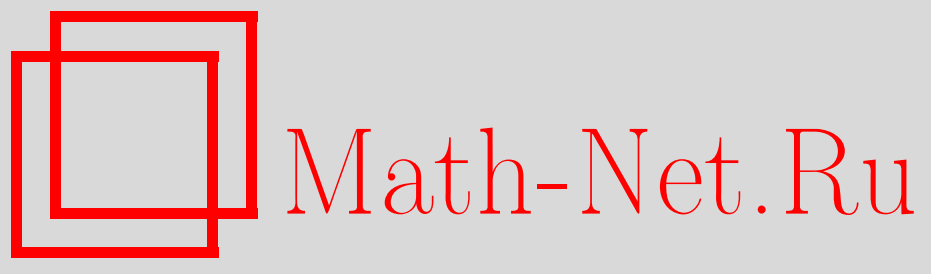

Н. В. Клыкова, Предельное распределение числа совпадающих промежутков, Теория вероятн. и ее примен., 2002, том 47, выпуск 1, 147-152

DOI: https://doi.org/10.4213/tvp3069

Использование Общероссийского математического портала MathNet.Ru подразумевает, что вы прочитали и согласны с пользовательским соглашением

http://www . mathnet .ru/rus/agreement

Параметры загрузки:

IP : 54.237 .206 .68

26 апреля 2023 г., 09:25:48

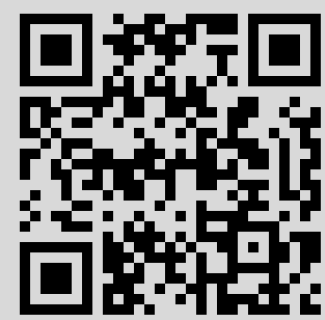


(C) $2002 \mathrm{r}$

КЛЫКОВА Н. В.*

\section{ПРЕДЕЛЬНОЕ РАСПРЕДЕЛЕНИЕ ЧИСЛА СОВПАДАЮЩИХ ПРОМЕЖУТКОВ}

Пусть $X_{1}, \ldots, X_{T}$ - независимые случайные величины, равномерно распределенные на множестве $\{1, \ldots, N\}$, и $X_{(1)} \leqslant X_{(2)} \leqslant \cdots \leqslant X_{(T)}$ построенный по ним вариационный ряд, а $\zeta(T, N)$ - число таких пар $(i, j), 1 \leqslant i<j \leqslant T-1$, что $X_{(i+1)}-X_{(i)}=X_{(j+1)}-X_{(j)}$. Приводится полное доказательство теоремы о сходимости распределения $\zeta(T, N)$ к распределению Пуассона с параметром $\lambda$ при $T, N \rightarrow \infty, T^{3} / 4 N \rightarrow \lambda$. Эвристическое обоснование этого утверждения было приведено в книге Олцуса [2].

Ключевые слова и фразы: задача о днях рождения, вариационный ряд, спейсинги.

Классическую задачу о днях рождения можно математически сформулировать как задачу о распределении числа пар совпадающих исходов в $T$ испытаниях по равновероятной полиномиальной схеме с $N$ исходами. Хорошо известно (см., например, [1]), что если $N, T \rightarrow \infty$ так, что $T^{2} / 2 N \rightarrow \lambda \in(0, \infty)$, то распределение числа таких пар сходится к пуассоновскому распределению с параметром $\lambda$.

В книге Олдуса [2] в виде задачи Е6 формулируется предложенный Дж. Марсалья критерий проверки качества датчиков псевдослучайных чисел, который состоит в следующем. Будем считать, что последовательность псевдослучайных чисел представляет собой последовательность $X_{1}, \ldots, X_{T}$ независимых случайных величин, равномерно распределенных на множестве $\{1, \ldots, N\}$. Построим по $X_{1}, \ldots, X_{T}$ вариационный ряд $X_{(1)} \leqslant X_{(2)} \leqslant \cdots \leqslant X_{(T)}$ и рассмотрим разности $\Delta_{k}=X_{(k+1)}-X_{(k)}$, $k=1, \ldots, T-1$. В качестве новой характеристики качества датчика псевдослучайных чисел Марсалья предложил использовать число $\zeta(T, N)$ таких пар $(i, j)$, $i \leqslant i<j \leqslant T-1$, что $\Delta_{i}=\Delta_{j}$. В [2] с помощью эвристических способов исследования предельных распределений сумм зависимых индикаторов (которым и посвящена эта книга) обоснована естественность пуассоновской аппроксимации для распределения $\zeta(T, N)$, когда $T, N \rightarrow \infty$ так, что $T^{3} / 4 N \rightarrow \lambda \in(0, \infty)$. Цель настояшей работы состоит в строгом доказательстве этого утверждения.

Tеорема. Если $T^{3} / 4 N \rightarrow \lambda \in(0, \infty)$ nрu $N \rightarrow \infty$, mo

$$
\lim _{N \rightarrow \infty} \mathbf{P}\{\zeta(T, N)=k\}=\frac{\lambda^{k}}{k !} e^{-\lambda}, \quad k=0,1 \ldots
$$

Следствие. Пусть $\tau_{N}=\min \{T: \zeta(T, N)>0\}$. Тогда

$$
\lim _{N \rightarrow \infty} \mathbf{P}\left\{\tau_{N} \geqslant x(4 N)^{1 / 3}\right\}=e^{-x^{3}}, \quad x \geqslant 0 .
$$

Д о к а 3 а т е л ь с т в о т е о р е м ы. Для троек $d=(r, u, v), 0 \leqslant r<N / 2$, $1 \leqslant u \leqslant u+r \leqslant v \leqslant N-r$, введем события

$$
A(r, u, v)=\bigcup_{1 \leqslant m<n<T}\left\{X_{(m)}=u, X_{(m+1)}=u+r, X_{(n)}=v, X_{(n+1)}=v+r\right\}
$$

и их индикаторы $\chi(d)=\chi(r, u, v)$. Положим

$$
\begin{aligned}
\zeta(T, N) & =\sum_{r=0}^{[(N-1) / 2]} \sum_{u=1}^{N-2 r} \sum_{v=u+r}^{N-r} \chi(r, u, v), \\
\zeta_{R}(T, N) & =\sum_{r=1}^{R} \sum_{u=1}^{N-2 r-1} \sum_{v=u+r+1}^{N-r} \chi(r, u, v)=\sum_{B} \chi(d),
\end{aligned}
$$

* Московский государственный университет им. М. В. Ломоносова, механико-математический факультет, Воробьевы горы, 119899, ГСП-1, Москва, Россия. 
где $B-$ множество всех троек $d=(r, u, v)$, образующих область суммирования тройной суммы в средней части (4).

Лемма 1. Если $R=R(T, N)$ изменяется так, что $N=o(R T) n p u T^{3} / 4 N \rightarrow$ $\lambda \in(0, \infty), N \rightarrow \infty, m o$

$$
\mathbf{E} \zeta_{R}(T, N) \rightarrow \lambda, \quad \mathbf{E} \zeta(T, N) \rightarrow \lambda \quad u \quad \mathbf{P}\left\{\zeta_{R}(T, N)=\zeta(T, N)\right\} \rightarrow 1
$$

Д о к а з а т е л ь с т в о. 1) Сначала получим оценки и асимптотические формулы для $\mathbf{P}\{\chi(r, u, v)=1\}$. Пусть $M(r, u, v)=\{u, u+r, v+r\}, m(r, u, v)$ обозначает число различных элементов множества $M(r, u, v)$, а $M^{*}(r, u, v)=\{j: u+1 \leqslant j \leqslant$ $u+r-1\} \cup\{j: v+1 \leqslant j \leqslant v+r-1\}$. Если $r \neq 0$, то

$$
\begin{aligned}
& \mathbf{P}\{\chi(r, u, v)=1\}=\mathbf{P}(A(r, u, v)) \\
& \quad=\mathbf{P}\left\{\exists 1 \leqslant m<n<T: X_{(m)}=u, X_{(m+1)}=u+r, X_{(n)}=v, X_{(n+1)}=v+r\right\} \\
& =\mathbf{P}\left\{M(r, u, v) \subset\left\{X_{1}, \ldots, X_{T}\right\}, M^{*}(r, u, v) \cap\left\{X_{1}, \ldots, X_{T}\right\}=\varnothing\right\} .
\end{aligned}
$$

Эту вероятность можно интерпретировать следующим образом. Пусть $T$ частиц независимо по равновероятной схеме размещаются по $N$ ячейкам $\{1, \ldots, N\}$, $\mu_{0}(T, N)$ - число пустых ячеек, а $\eta_{j}(T, N)$ - число частиц, попавших в $j$-ю ячейку. Тогда

$$
A(r, u, v)=\left\{\eta_{j}(T, N)>0 \forall j \in M(r, u, v), \eta_{j}(T, N)=0 \forall j \in M^{*}(r, u, v)\right\} .
$$

Используя формулу полной вероятности по числу частиц, попавших в ячейки из множества $M(r, u, v)$, находим, что при $N, T \rightarrow \infty, T^{3}=O(N), r>0$

$$
\begin{aligned}
& \mathbf{P}(A(r, u, v))= \sum_{n=m(r, u, v)}^{T} C_{T}^{n} m(r, u, v)^{n}(N-2(r-1)-m(r, u, v))^{T-n} N^{-T} \\
& \times \mathbf{P}\left\{\mu_{0}(n, m(r, u, v))=0\right\} \\
&=(1+o(1)) C_{T}^{m(r, u, v)}(N-2 r)^{T-m(r, u, v)} m(r, u, v)^{m(r, u, v)} N^{-T} \\
& \times \mathbf{P}\left\{\mu_{0}(m(r, u, v), m(r, u, v))=0\right\} \\
&=(1+o(1)) C_{T}^{m(r, u, v)}(N-2 r)^{T-m(r, u, v)} m(r, u, v) ! N^{-T} \\
&=(1+o(1)) T^{m(r, u, v)}(N-2 r)^{T-m(r, u, v)} N^{-T} .
\end{aligned}
$$

При $r=0$ и $u \neq v$

$$
\begin{aligned}
\mathbf{P}(A(0, u, v)) & =\sum_{n=4}^{T} C_{T}^{n} 2^{n}(N-2)^{T-n} N^{-T} \mathbf{P}\left\{\eta_{1}(n, 2) \geqslant 2, \eta_{2}(n, 2) \geqslant 2\right\} \\
& <16 C_{T}^{4} N^{-4} \frac{1}{1-2 T / 5 N} .
\end{aligned}
$$

Если $r=0$ и $u=v$, то

$$
\mathbf{P}(A(0, u, u))=\sum_{n=3}^{T} C_{T}^{n}(N-1)^{T-n} N^{-T}<C_{T}^{3} N^{-3} \frac{1}{1-T / 4 N} .
$$

2) Покажем теперь, что в условиях леммы

$$
\mathbf{E} \zeta(T, N)=\sum_{r=0}^{[(N-1) / 2]} \sum_{u=1}^{N-2 r} \sum_{v=u+r}^{N-r} \mathbf{P}\{\chi(r, u, v)=1\} \rightarrow \lambda \quad \text { и } \quad \mathbf{E} \zeta_{R}(T, N) \rightarrow \lambda .
$$


a) Вклад в $\mathbf{E} \zeta(T, N)$ слагаемых, для которых $m(r, u, v)=4$ (т.е. $r \neq 0$ и $u+r \neq v)$, при $N, T \rightarrow \infty, T^{3}=O(N)$ составляет

$$
\begin{aligned}
& \sum_{r=1}^{[(N-2) / 2]} \sum_{u=1}^{N-2 r-1} \sum_{v=u+r+1}^{N-2 r} \mathbf{P}\{\chi(r, u, v)=1\} \\
& =(1+o(1)) \sum_{r=1}^{[(N-2) / 2]} \sum_{u=1}^{N-2 r-1} T^{4} N^{-T}(N-2 r)^{T-4}(N-2 r-u) \\
& =(1+o(1)) T^{4} N^{-T} \sum_{r=1}^{[(N-2) / 2]}(N-2 r)^{T-4}(N-2 r) \frac{N-2 r-1}{2} \\
& =(1+o(1)) \frac{1}{2} T^{4} N^{-T} \sum_{r=0}^{N / 2}(N-2 r)^{T-2}=(1+o(1)) \frac{T^{3}}{4 N} \rightarrow \lambda .
\end{aligned}
$$

Заметим, что при $N, T, R \rightarrow \infty, T^{3}=O(N)$

$$
2 \sum_{r=1}^{R}(N-2 r)^{T-2}=(1+o(1)) \frac{N^{T-1}}{T-1}\left(1-\left(1-\frac{2 R}{N}\right)^{T-1}\right) .
$$

Если $N=o(R T)$, то $(1-2 R / N)^{T-1} \rightarrow 0$ в условиях леммы. Тогда при $N, T \rightarrow \infty$, $T^{3}=O(N), N=o(R T)$

$$
\begin{aligned}
\mathbf{E} \zeta_{R}(T, N) & =\sum_{r=1}^{R} \sum_{u=1}^{N-2 r-1} \sum_{v=u+r+1}^{N-r} \mathbf{P}\{\chi(r, u, v)=1\} \\
& =(1+o(1)) \frac{T^{3}}{4 N}\left(1-\left(1-\frac{2 R}{N}\right)^{T-1}\right) \rightarrow \frac{T^{3}}{4 N} \rightarrow \lambda .
\end{aligned}
$$

Первое утверждение леммы доказано.

б) При $N, T \rightarrow \infty, T^{3}=O(N)$ вклад в $\mathbf{E} \zeta(T, N)$ слагаемых, для которых $r \neq 0$ и $m(r, u, v)=3$, составляет

$$
(1+o(1)) \sum_{r=1}^{[(N-1) / 2]} \sum_{u=1}^{N-2 r} N^{-T} C_{T}^{3} 3 !(N-2 r-1)^{T-3}=(1+o(1)) \frac{T^{2}}{2 N}=o(1),
$$

вклад слагаемых, для которых $r=0, u \neq v$, меньше

$$
(1+o(1)) \sum_{u=1}^{N-1} \sum_{v=u+1}^{N} T^{4} N^{-4} \frac{1}{1-2 T / 5 N}=O\left(\frac{T^{4}}{N^{2}}\right)=o(1)
$$

и вклад слагаемых, для которых $r=0, u=v$, меньше

$$
(1+o(1)) \sum_{u=1}^{N} C_{T}^{3} N^{-3} \frac{1}{1-T / 4 N}=O\left(\frac{T^{3}}{N^{2}}\right)=o(1) .
$$

Из а), б) следует, что существенное влияние на асимптотическое поведение $\mathbf{E} \zeta(T, N)$ оказывают лишь слагаемые из п. а), следовательно, $\mathbf{E} \zeta(T, N) \rightarrow \lambda$ в условиях леммы.

3) Соотношение $\mathbf{P}\left\{\zeta_{R}(T, N)=\zeta(T, N)\right\} \rightarrow 1$ следует из того, что $\mathbf{P}\left\{\zeta_{R}(T, N) \leqslant\right.$ $\zeta(T, N)\}=1$, а пределы $\mathbf{E} \zeta(T, N)$ и $\mathbf{E} \zeta_{R}(T, N)$ совпадают

$$
\mathbf{P}\left\{\zeta_{R}(T, N) \neq \zeta(T, N)\right\} \leqslant \mathbf{E}\left(\zeta(T, N)-\zeta_{R}(T, N)\right)=\mathbf{E} \zeta(T, N)-\mathbf{E} \zeta_{R}(T, N)=o(1) .
$$

Лемма 1 доказана.

В силу леммы 1 теорему достаточно доказать для случайных величин $\zeta_{R}(T, N)$. Для этого воспользуемся методом моментов в форме, предложенной Б. А. Севастьяновым [3]. Для доказательства сходимости факториальных моментов $m_{k}=$ $\mathbf{E}\left(\zeta_{R}(T, N)\right)^{[k]}$ к $\lambda^{k}$ при $N, T \rightarrow \infty$ достаточно показать, что $\mathbf{E} \zeta_{R}(T, N) \rightarrow \lambda$ и что 
для каждого $k=2,3, \ldots$ можно указать исключительные множества $I_{k} \subset B^{k}$, состоящие из упорядоченных попарно различных наборов $\left(d_{1}, \ldots, d_{k}\right)$, где $d_{i}=\left(r_{i}, u_{i}, v_{i}\right)$, $i=1, \ldots, k$, и удовлетворяющие следующим условиям:

$$
\begin{array}{r}
\lim _{N, T \rightarrow \infty} \sum_{I_{k}} \prod_{i=1}^{k} \mathbf{P}\left(A\left(d_{i}\right)\right)=\lim _{N, T \rightarrow \infty} \sum_{I_{k}} \mathbf{P}\left\{\bigcap_{i=1}^{k} A\left(d_{i}\right)\right\}=0, \\
\lim _{N, T \rightarrow \infty} \max _{d_{i} \notin I_{k}}\left|\frac{\mathbf{P}\left(A\left(d_{1}\right), \ldots, A\left(d_{k}\right)\right)}{\mathbf{P}\left(A\left(d_{1}\right)\right) \cdots \mathbf{P}\left(A\left(d_{k}\right)\right)}-1\right|=0 .
\end{array}
$$

Для набора $\left(d_{1}, \ldots, d_{k}\right) \subset B^{k}$, где $d_{i}=\left(r_{i}, u_{i}, v_{i}\right), i=1, \ldots, k$, введем обозначение $m\left(d_{1}, \ldots, d_{k}\right)=\left|\cup_{i=1}^{k} M\left(r_{i}, u_{i}, v_{i}\right)\right|$. Положим

$$
I_{k}=I_{k}^{1} \cup I_{k}^{2}
$$

где

$$
\begin{aligned}
& I_{k}^{1}=\left\{\left(d_{1}, \ldots, d_{k}\right) \in B^{k}: \mathbf{P}\left(A\left(d_{1}\right), \ldots, A\left(d_{k}\right)\right)=0\right\} \\
& I_{k}^{2}=\left\{\left(d_{1}, \ldots, d_{k}\right) \in B^{k}: m\left(d_{1}, \ldots, d_{k}\right) \neq 4 k\right\} .
\end{aligned}
$$

Лемма 2. Если множества $I_{k}$ выбраны в соответствии с формулой (7), то соотночения (5) $и$ (6) выполняются.

Д о к а з а т е л ь с т в о. Легко проверить, что

$$
\begin{aligned}
\mathbf{P}\left\{\bigcap_{i=1}^{k} A\left(d_{i}\right)\right\}= & \mathbf{P}\left\{\bigcup_{i=1}^{k} M\left(r_{i}, u_{i}, v_{i}\right) \subset\left\{X_{1}, \ldots, X_{T}\right\},\right. \\
& \left.\bigcup_{i=1}^{k} M^{*}\left(r_{i}, u_{i}, v_{i}\right) \cap\left\{X_{1}, \ldots, X_{T}\right\}=\varnothing\right\} .
\end{aligned}
$$

Используем такую же интерпретацию, как в п. 1) доказательства леммы 1 , и применим формулу полной вероятности по числу частиц, попавших в ячейки из множества $\cup_{i=1}^{k} M\left(r_{i}, u_{i}, v_{i}\right)$. Если $d_{i} \notin I_{k}^{1}$, т.е. $\cup_{i=1}^{k} M\left(r_{i}, u_{i}, v_{i}\right) \cap \cup_{i=1}^{k} M^{*}\left(r_{i}, u_{i}, v_{i}\right)=\varnothing$, то при $k=$ const $, N, T \rightarrow \infty, T^{3}=O(N)$

$$
\begin{aligned}
\mathbf{P} & \left\{\bigcap_{i=1}^{k} A\left(d_{i}\right)\right\} \\
& =(1+o(1)) N^{-T} C_{T}^{m\left(d_{1}, \ldots, d_{k}\right)} m\left(d_{1}, \ldots, d_{k}\right) !\left(N-2\left(r_{1}+\cdots+r_{k}\right)\right)^{T-m\left(d_{1}, \ldots, d_{k}\right)} \\
& =(1+o(1)) N^{-T} T^{m\left(d_{1}, \ldots, d_{k}\right)}\left(N-2\left(r_{1}+\cdots+r_{k}\right)\right)^{T-m\left(d_{1}, \ldots, d_{k}\right)} .
\end{aligned}
$$

Если $d_{i} \in I_{k}^{1}$, то $\mathbf{P}\left\{\cap_{i=1}^{k} A\left(d_{i}\right)\right\}=0$, причем в силу определения величины $\zeta_{R}(T, N)$

$$
\mathbf{P}\left(A\left(d_{i}\right)\right)=(1+o(1)) N^{-T} T^{4}\left(N-2 r_{i}\right)^{T-4} .
$$

2) Покажем теперь, что выполняется соотношение (6). Пусть $\left(d_{1}, \ldots, d_{k}\right) \notin I_{k}$, тогда, используя соотношения (8) и (9), получим при $k=$ const, $N, T \rightarrow \infty, T^{3}=O(N)$

$$
\begin{aligned}
\frac{\mathbf{P}\left(A\left(d_{1}\right), \ldots, A\left(d_{k}\right)\right)}{\mathbf{P}\left(A\left(d_{1}\right)\right) \cdots \mathbf{P}\left(A\left(d_{k}\right)\right)} & =(1+o(1)) \frac{\left(N-2\left(r_{1}+\cdots+r_{k}\right)\right)^{T-4 k} N^{T k}}{N^{T}\left(N-2 r_{1}\right)^{T-4} \cdots\left(N-2 r_{k}\right)^{T-4}} \\
& =(1+o(1)) \frac{\left(1-2\left(r_{1}+\cdots+r_{k}\right) / N\right)^{T-4 k}}{\prod_{i=1}^{k}\left(1-2 r_{i} / N\right)^{T-4}} .
\end{aligned}
$$

Возьмем для простоты доказательства $R=T^{9 / 4}$, тогда $N=o(R T)$ и $r_{1}+\cdots+r_{k}=$ $O(R)$ при $N, T \rightarrow \infty, T^{3}=O(N)$, поэтому

$$
\begin{aligned}
\ln \frac{\mathbf{P}\left(A\left(d_{1}\right), \ldots, A\left(d_{k}\right)\right)}{\mathbf{P}\left(A\left(d_{1}\right)\right) \cdots \mathbf{P}\left(A\left(d_{k}\right)\right)}= & (T-4) \frac{2\left(r_{1}+\cdots+r_{k}\right)}{N}-(T-4 k) \frac{2\left(r_{1}+\cdots+r_{k}\right)}{N} \\
& +O\left(\frac{R}{N}+T \frac{R^{2}}{N^{2}}\right)=O\left(N^{-1 / 6}\right)=o(1),
\end{aligned}
$$

следовательно, (6) выполняется. 
3) Покажем сначала, что соотношения (5) выполняются для множества $I_{k}^{2} \subset I_{k}$. Так как набор $\left(d_{1}, \ldots, d_{k}\right) \in I_{k}^{2}$, если $m\left(d_{1}, \ldots, d_{k}\right) \neq 4 k$, то существуют такие $i \neq j$, что $M\left(r_{i}, u_{i}, v_{i}\right) \cap M\left(r_{j}, u_{j}, v_{j}\right) \neq \varnothing$.

a) При $N, T \rightarrow \infty, T^{3}=O(N), k=$ const

$$
\begin{aligned}
& \sum_{I_{k}^{2}} \prod_{i=1}^{k} \mathbf{P}\left(A\left(d_{i}\right)\right) \leqslant C_{k}^{2} \sum^{*} \mathbf{P}\left(A\left(d_{1}\right)\right) \mathbf{P}\left(A\left(d_{2}\right)\right)\left(\sum_{d} \mathbf{P}(A(d))\right)^{k-2} \\
& =C_{k}^{2} \sum^{*} \mathbf{P}\left(A\left(d_{1}\right)\right) \mathbf{P}\left(A\left(d_{2}\right)\right) \lambda^{k-1} \\
& =O\left(\sum_{r_{1}, r_{2}=0}^{N / 2} N^{-2 T} T^{8}\left(N-2 r_{1}\right)^{T-4}\left(N-2 r_{2}\right)^{T-4} N^{3}\right)=O\left(N^{-1}\right)=o(1),
\end{aligned}
$$

где суммирование в $\sum^{*}$ ведется по $d_{1}, d_{2}$ таким, что $M\left(d_{1}\right) \cap M\left(d_{2}\right) \neq \varnothing$.

б) Используя формулу (8), находим:

$$
\begin{aligned}
\sum_{I_{k}^{2}} \mathbf{P}\left\{\bigcap_{i=1}^{k} A\left(d_{i}\right)\right\} & \leqslant \sum_{l=1}^{4 k-1} \sum^{\prime} \mathbf{P}\left\{\bigcap_{i=1}^{k} A\left(d_{i}\right)\right\} \\
& \leqslant \sum_{l=1}^{4 k-1} \sum^{\prime} N^{-T} T^{4 k-l}\left(N-2\left(r_{1}+\cdots+r_{k}\right)\right)^{T-4 k+l} \\
& =O\left(\sum_{l=1}^{4 k-1} \sum^{\prime \prime} T^{4 k-l} N^{-2 k}\left(1-\frac{2\left(r_{1}+\cdots+r_{k}\right)}{N}\right)^{T}\right) \\
& =O\left(\sum_{l=1}^{4 k-1} \sum^{\prime \prime} T^{4 k-l} N^{-2 k}\left(1-\frac{1}{N}\right)^{2 T \sum_{i=1}^{k} r_{i}}\right),
\end{aligned}
$$

где сумма $\sum^{\prime}$ берется по $\left(d_{1}, \ldots, d_{k}\right)$ таким, что $m\left(d_{1}, \ldots, d_{k}\right)=4 k-l$, а сумма $\sum^{\prime \prime}-$ по $r_{1}, \ldots, r_{k} \geqslant 0$ таким, что $r_{1}+\cdots+r_{k}<N / 2$. Заметим, что

$$
\sum^{\prime \prime}\left(1-\frac{1}{N}\right)^{2 T \sum_{i=1}^{k} r_{i}} \leqslant\left(\sum_{r=0}^{\infty}\left(1-\frac{1}{N}\right)^{2 T r}\right)^{k}<\left(\frac{N}{T}\right)^{k} .
$$

Используя неравенство (10), получим:

$$
\sum_{I_{k}^{2}} \mathbf{P}\left\{\bigcap_{i=1}^{k} A\left(d_{i}\right)\right\}=O\left(\sum_{l=1}^{4 k-1} T^{4 k-1} N^{-2 k} \frac{N^{k}}{T^{k}}\right)=O\left(\frac{1}{T}\right)=o(1) .
$$

Из пाг. а), б) следует, что для множества $I_{k}^{2} \subset I_{k}$ соотношения (5) выполняются.

4) Осталось показать, что (5) выполняется для множества $I_{k}^{1} \subset I_{k}$, т.е., что $\lim _{N, T \rightarrow \infty} \sum_{I_{k}^{1}} \prod_{i=1}^{k} \mathbf{P}\left(A\left(d_{i}\right)\right)=0 . \quad$ Если $\left(d_{1}, \ldots, d_{k}\right) \in I_{k}^{1}$, то $M\left(r_{i}, u_{i}, v_{i}\right) \cap$ $\left(\cup_{j \neq i} M^{*}\left(r_{j}, u_{j}, v_{j}\right)\right) \neq \varnothing$ при некотором $i$, т.е. $\left\{u_{i}, v_{i}\right\} \cap\left(\cup_{j \neq i}\left(\left[u_{j}-r_{j}+1, u_{j}+r_{j}-1\right] \cup\right.\right.$ $\left.\left.\left[v_{j}-r_{j}+1, v_{j}+r_{j}-1\right]\right)\right) \neq \varnothing$. Заметим, что при $N, T \rightarrow \infty, T^{3}=O(N)$

$$
\sum_{r=0}^{N / 2} r(N-2 r)^{T-4}=2^{T-4} \sum_{r=0}^{N / 2} r^{T-4}\left(\frac{N}{2}-r\right)=O\left(N^{T-2} T^{-2}\right) .
$$

Используя формулы (9) и (11), при $N, T \rightarrow \infty, T^{3}=O(N), k=$ const находим:

$$
\begin{aligned}
& \sum_{\left(d_{1}, \ldots, d_{k}\right) \in I_{k}^{1}} \mathbf{P}\left(A\left(d_{1}\right)\right) \cdots \mathbf{P}\left(A\left(d_{k}\right)\right) \\
& \leqslant \sum_{i=1}^{k} \sum_{d_{j}: j \in\{1, \ldots, k\} \backslash\{i\} d_{i}: M\left(d_{i}\right) \cap\left(\cup_{j \neq i} M^{*}\left(d_{j}\right)\right) \neq \varnothing} \prod_{m=1}^{k} \mathbf{P}\left(A\left(d_{m}\right)\right) \\
& =O\left(T^{4 k} N^{-k T} N^{2(k-1)} \sum_{i=1}^{k} N^{3} \sum_{r_{1}, \ldots, r_{k}=0}^{N / 2}\left(r_{1}+\cdots+r_{k}+k r_{i}\right) \prod_{j=1}^{k}\left(N-2 r_{j}\right)^{T-4}\right)
\end{aligned}
$$




$$
\begin{aligned}
& =O\left(T^{4 k} N^{-k T} N^{2(k+1)}\left(\frac{N^{T-3}}{T}\right)^{k-1} N^{T-2} T^{-2}\right) \\
& =O\left(\left(\frac{T}{N}\right)^{k} T^{-1}\right)=O\left(T^{-1}\right)=o(1) .
\end{aligned}
$$

Соотношение (5) и тем самым лемма 2 доказаны.

В силу леммы 2 для случайной величины $\zeta_{R}(T, N)$ выполнены условия теоремы Б. А. Севастьянова, следовательно, $\zeta_{R}(T, N)$ (а значит, в силу леммы 1 и $\zeta(T, N)$ ) имеет в пределе пуассоновское распределение с параметром $\lambda$. Теорема доказана.

\section{СПИСОК ЈИТЕРАТУРЫ}

1. Колчин В.Ф., Севастьянов Б.А., Чистяков В.П. Случайные размещения. М.: Наука, 1976, 223 c.

2. Aldous D. Probability Approximation via the Poisson Clumping Heuristic. BerlinHeidelberg: Springer-Verlag, 1989, 269 p.

3. Севастьянов Б. А. Предельный закон Пуассона в схеме сумм зависимых случайных величин. - Теория вероятн. и ее примен., 1972, т. 17, в. 4, с. 733-738.

Поступила в редакцию 12.III.2001

(c) $2002 \Gamma$.

КУКСИН С. Б., НАДИРАШВИЛИ Н. С. ${ }^{* *}$, ПЯТНИЦКИЙ А. Л.***

\section{ОЦЕНКИ ГЁЛЬДЕРОВСКИХ НОРМ ДЛЯ РЕШЕНИЙ СТОХАСТИЧЕСКИХ УРАВНЕНИЙ В ЧАСТНЫХ ПРОИЗВОДНЫХ}

В работе рассмотрены стохастические уравнения с частными производными второго порядка с аддитивным шумом, заданные в ограниченной области пространства $\mathbf{R}^{d}$. В предположении, что коэффициенты при шуме являются $L^{p}$-функциями с достаточно большим $p$, мы доказываем, что решения непрерывны по Гёльдеру п.н., и что соответствующие гёльдеровские нормы имеют конечные моменты любого порядка.

Ключевые слова и фразы: стохастические уравнения, непрерывность по Гёльдеру.

* Heriot-Watt University, Edinburgh, EH14 4AS, UK; Математический институт им. В. А. Стеклова РАН, ул. Губкина, 8, 117966 Москва, ГСП-1, Россия; е-mail: S.B.Kuksin@ma.hw.ac.uk

** University of Chicago, Department of Mathematics, 5734 University Avenue, Chicago, IL 60637, U.S.A.; e-mail: nicholas@math.uchicago.edu

*** Narvik University College, HiN, Postbox 385, 8505, Narvik, Norway; Физический институт им. П. Н. Лебедева РАН, Ленинский проспект, 53, 117333 Москва, Россия; e-mail: andrey@sci.lebedev.ru 